\title{
La Educación Médica en tiempos de pandemia COVID-19: Reflexiones de la Red de Educación Médica y Ciencias de la Salud de Chile
}

\author{
Medical Education in times of the Covid-19 Pandemic: Reflections of the Red de \\ Educación Médica y Ciencias de la Salud de Chile
}

\author{
Lucía Santelices Cuevas' ${ }^{1}$ María Guerra Zúñiga² y Giselle Myer ${ }^{3}$
}

\section{Resumen}

El objetivo del ensayo es realizar un análisis de las variables implicadas en el proceso educativo en las carreras de las Ciencias de la Salud en Chile durante la pandemia por COVID, que desde la perspectiva de las autoras merecen una atención particular. Se consideró 3 aspectos: la selección de contenidos de aprendizaje, la utilización de la tecnología de parte de los docentes y de los estudiantes y la modificación de un sistema de evaluación tradicional por un sistema de evaluación auténtica. Se finaliza el ensayo compartiendo las estrategias con las que diversas Escuelas de Medicina del país enfrentaron la emergencia.

Palabras clave: educación médica; Coronavirus; curriculum; aprendizaje; evaluación.

\begin{abstract}
The objective of this essay is to carry out an analysis of the variables involved in the educational process in Health Sciences careers in Chile during the COVID pandemic, which from the authors' perspective deserve particular attention. Three aspects were considered: the selection of learning contents, the use of technology by teachers and students, and the modification of a traditional evaluation system for an authentic evaluation system. The essay ends by sharing the strategies with which various medical schools in the country faced the health emergency.
\end{abstract}

Keywords: Medical Education; Coronavirus; curriculum; learning; assessment.

Fecha de envío: 2021-09-12 - Fecha de aprobación: 2021-12-06

Parece importante que después de un año de urgencias y ajustes realizados a la Educación Médica y Ciencias de la Salud, detenerse y analizar algunos aspectos que exigen ser observados con detención para recoger los frutos que esta circunstancia ha dejado en la docencia universitaria.

Un aspecto se refiere, a la necesidad de asumir la docencia y transformarla desde entornos presenciales a entornos online. Con lo cual se debieron ajustar tres aspectos: el conocimiento, la evaluación y la utilización de la tecnología.

El conocimiento necesariamente tuvo que ser reorganizado y se planteó la necesidad de cambiar la concepción de transmitir gran cantidad de información por la selección de lo primordial del saber, para complementarlo con actividades centradas en la búsqueda de información y su aplicación por parte de los estudiantes.
Por otra parte, un segundo aspecto que exigió revisión, fue la evaluación de los aprendizajes. Por años, la instancia más importante para controlar el aprendizaje estuvo centrada en las pruebas de alta ponderación. Sin embargo, la necesidad de cerciorarse acerca del avance del aprendizaje, impulsó el cambio desde una evaluación vigente por años a un sistema de evaluación auténtica (Reyes Garcia et al., 2013). Con este cambio adquirieron relevancia otras formas de instrumentos de evaluación y con ello un avance hacia la autorregulación del aprendizaje.

El tercer aspecto que experimentó una anhelada transformación en la docencia fue el uso de la tecnología, que obligó a docentes y estudiantes a emprender nuevas formas de implementar los ciclos de aprendizaje. Las clases presenciales se debieron ajustar a espacios más breves, de interacción directa, lo que llevó a transformar las sesiones desde un centro en la información relatada por el docente,

(1) Centro de Investigación en Educación Médica y Ciencias de la Salud, Universidad Finis Terrae, Santiago. Chile.

(2) Oficina de Educación Médica, Universidad de Valparaíso, Valparaíso. Chile.

(3) Oficina de Educación Médica, Universidad Católica del Norte, Coquimbo. Chile.

Autor de correspondencia: maria.guerra@uv.cl 
a un intercambio de información y colaboración para articular los conocimientos de los docentes con las actividades asincrónicas realizadas por los estudiantes.

Lo señalado, con una nueva forma telemática de hacer docencia permitió trabajar y descubrir nuevas posibilidades en la docencia en ciencias de la salud que ya habían sido descritas por Jason (2002) y que se pueden resumir en cuatro aspectos:

- Una organización menos definida del espacio y el tiempo

- Mayor uso de las tecnologías de información

- Una organización del aprendizaje menos guiada y

- Materiales de aprendizaje con una base tecnológica mayor.

Lo anterior no fue lo único que se modificó. También lo hizo la gestión del aprendizaje. No había espacios para explicar los contenidos y se optó, en general por aportar en los espacios sincrónicos, el manejo conceptual básico y entregar a los estudiantes las herramientas para indagar en redes de internet especializadas, con el fin, de estimular la aplicación del saber. Ello trajo consigo modificar el diseño de actividades.

Se privilegiaron actividades que impulsaron a los estudiantes a buscar y seleccionar documentos e información relevante, habilidades para convertir información en conocimiento y habilidades para comunicar conocimiento.

Se adelantó una modificación que se avizoraba desde el año 2010 por Reyes-García et al. (2013) quienes presagiaron esta modificación de la docencia en educación superior para finales del siglo XX. Se planteaba que el aprendizaje sería más ubicuo y móvil gracias a los dispositivos digitales (Vázquez-Cano \& Sevillano, 2015) y que se modificaría un consumo individual de información por una construcción colaborativa y conjunta del conocimiento.

Durante la pandemia, lo que se señalaba para un futuro se hizo realidad en Chile y otros países latinoamericanos con más prontitud.

Los educadores en ciencias de la salud se organizaron y se inició un conjunto de webinars y reuniones científicas, que dieron lugar a un valioso intercambio de conocimientos y experiencias.

\section{Las experiencias compartidas}

En primer lugar se centraron en los estudiantes y se pueden sacar algunas conclusiones preliminares:

Los estudiantes exigieron atención particular desde tres perspectivas. Desde la perspectiva psico-afectiva, porque ellos utilizan la tecnología en su vida cotidiana con sus pares, no obstante, no tienen las competencias académicas para aprender con la tecnología, no están habituados a atender ni a responder a los requerimientos de los docentes en escenarios online. Se suma a lo anterior que muchos tuvieron que ser apoyados institucionalmente con los dispositivos tecnológicos, bolsas de internet y utilización de nuevas plataformas para un trabajo académico online.

En segundo lugar, se rompió la idea de que el aprendizaje se encuentra en los contenidos y se comenzó una discusión e intercambios respecto de las actividades que se deberían realizar para que ellos pudiesen ser comprendidos por los estudiantes y facilitar de esa forma el acceso a la información.

En tercer lugar, surgió la inquietud acerca de la evaluación y la necesidad de constatar los aprendizajes alcanzados. Lo señalado resultaba muy pertinente por la relativa pérdida del control por parte de los docentes y porque estudios previos señalaban que el aumento del uso de la tecnología para aprender, no se condice con una mejoría en el rendimiento académico (Barrera-Osorio \& Linden, 2009).

También se incorporó a la discusión el hecho de que la tecnología podría traer beneficios. Se mencionó insistentemente que las herramientas cognitivas estimulan los procesos cognitivos, constituyendo el fundamento de la construcción del conocimiento. Hecho que estaba fundado en trabajos publicados con anterioridad y refrendaba la idea que, uno de los beneficios del uso de la tecnología es que los estudiantes descargan procesos cognitivos de bajo nivel para centrarse en tareas cognitivas más complejas, amplificando con ello la forma de aprender.

También, se puso foco en los docentes quienes, frente a los desafíos impuestos por la pandemia, tuvieron que adaptar la planificación de sus cursos a un modelo virtual. Diseñar actividades de aprendizaje en este contexto, significó para ellos modificar metodologías de enseñanza y elaborar instrumentos de evaluación aplicables en el modelo online, con criterios de calidad. Implementar actividades de retroalimentación en este nuevo escenario educativo también fue primordial.

Los docentes tuvieron que convertirse forzosamente en usuarios de plataformas institucionales, Zoom, Google Meet, Google Classroom y Team por poner algunos ejemplos.

Los docentes tuvieron que transitar desde una interacción directa con los estudiantes a una relación indirecta, virtual, siendo esto un desafío, especialmente para los docentes clínicos, quienes tuvieron que cambiar un ámbito clínico presencial a uno telemático, cumpliendo un papel dual, asistencial y académico. Las instituciones frente a estos retos, tuvieron que capacitar a sus docentes en como desarrollar sus asignaturas en un entorno virtual, en el uso de la 
tecnología de la información y la comunicación (TIC) y entrenar a los académicos en simulación. La simulación clínica a distancia obligatoriamente, tuvo que incluirse en las actividades curriculares.

Las universidades realizaron el esfuerzo por capacitar a sus docentes en la utilización de las plataformas a través de cursos voluntarios que se alojaron en la plataforma institucional y que fueron generados por la Vicerrectoría Académica.

En las Facultades de Medicina, las oficinas de Educación Médica y Ciencias de la Salud, abordaron el apoyo a los docentes a través de distintas vías y recursos.

En algunas se realizó previamente, una detección de necesidades de perfeccionamiento de los docentes y se respondió al diagnóstico de esta con el diseño de cápsulas que abordaron aspectos metodológicos para apoyar la gestión docente y atender los tiempos que cada académico destinaba a su propio aprendizaje.

En otras universidades se hizo un acompañamiento directo a los profesores con menos experiencia en tecnologías informáticas. Algunas, hicieron un catastro de todos aquellos docentes que tuvieran formación de postítulo o postgrado en docencia, ellos previa consulta, pasaron a aumentar el número de los académicos que conformaban la oficina de Educación Médica. Los docentes incorporados se distribuyeron por niveles desde primero a quinto año de la carrera, acompañando directamente a sus pares en: manejo de aula virtual, elaboración y revisión de pruebas virtuales y uso de TICs para dinamizar las clases.

Por otro lado, se iniciaron ciclos de reuniones con los coordinadores para identificar oportunamente alguna necesidad a propósito del contexto. En paralelo, respecto de los estudiantes, se realizaron reuniones periódicas con el Centro de Estudiantes, de tal forma de acoger y escuchar las necesidades del estudiantado y canalizar puntos de encuentro para el ejercicio de la docencia.

Otras unidades, llevaron a cabo cursos on-line al inicio de la pandemia, dirigido a los coordinadores y docentes de las asignaturas de primer semestre, en donde se les capacitó en planificación, metodología, instrumentos de evaluación y retroalimentación/feedback como conceptos generales, aprovechando la oportunidad de hacer una inmersión en el mundo educativo para posteriormente adaptar la conceptualización pedagógica al entorno virtual. Además, se realizó un acompañamiento personalizado por un miembro de la oficina Educación Médica, a cada coordinador para apoyarlo en el proceso. Este proceso se replicó en todas las asignaturas.

Pareció importante reflexionar también acerca del curriculum, conciliando los ajustes con la necesidad de alcanzar las metas programadas. Se rediseñaron los programas de cursos, cuidando que no se modificaran los logros de aprendizaje requeridos por los perfiles de egreso. Para ello, se ajustaron las unidades de aprendizaje. La primera tarea, fue adaptar los resultados de aprendizaje con sus correspondientes contenidos. Estos fueron abordados en espacios sincrónicos, asincrónicos y autónomos. Ello llevó a generar modificaciones en las metodologías de enseñanza con el fin de facilitar a los estudiantes el acceso a la información, seleccionada por el docente.

Los contenidos para trabajar en el espacio sincrónico se concretaron en clases breves, no más de 45 minutos, las que fueron alojadas en plataformas institucionales y otras gratuitas, como Youtube. Las clases quedaban a libre disposición de los estudiantes para acceder las veces que lo estimaran conveniente. Además se destinaron espacios sincrónicos de integración de conocimientos abordados en función de casos clínicos diseñados para aplicar los contenidos. En los espacios asincrónicos, los estudiantes pudieron recibir videos, guías y un sin número de recursos adicionales. Se develaron las redes de información especializada utilizadas por los expertos, para que los estudiantes pudiesen realizar búsquedas de información y solucionar problemas con la orientación de los docentes.

Los espacios de autonomía eran utilizados discrecionalmente por los estudiantes. Este hecho puso en evidencia problemas de autorregulación y autonomía de los estudiantes, no sabiendo estos limitar sus espacios personales y académicos. Esto confirma estudios que señalan que los alumnos están capacitados para usar las redes sociales en su vida diaria pero no para transferir esas conductas al trabajo universitario. (Valtonen et al., 2011)

En los procesos evaluativos, se tuvo que modificar los instrumentos e instancias evaluativas. Al respecto, inicialmente se produjo preocupación sobre la copia y plagio online, algunas casas superiores utilizaban diferentes métodos para reducir la incertidumbre, tales como, obligar a los estudiantes a encender las cámaras durante la evaluación, contratar servicios de proctoring, limitar el tiempo de duración del espacio para responder cada ítem de la prueba y elaborar pruebas de desarrollo o respuestas breves.

No obstante, lo señalado, deja un espacio para la reflexión, porque la evidencia existente no es suficiente para fundamentar los miedos de los docentes respecto de los logros de aprendizaje esperados, utilizando la evaluación online. Se señala que la copia o plagio en evaluaciones online no superaría a la que ocurre en una instancia presencial (Raman et al., 2021) pero no existe evidencia empírica suficiente en favor de una u otra visión.

La experiencia lograda deja más que alguna interrogante por resolver, sin embargo, el trabajo mancomunado y solidario de la 


\section{Santelices et al.}

Red de Educación Médica y Ciencias de la Salud de Chile, permitió valiosos aprendizajes que se constituyen hoy en un aporte para quienes tienen a su cargo la formación de profesionales de la salud.

\section{Contribuciones y reconocimientos}

Este trabajo no tuvo fuentes de financiamiento.

La contribución de la primera autora radica en la idea original y estructuracón de los pilares básicos de la reflexión en docencia. Segunda y tercera autoras aportan a la revisión del ensayo y discusión de los pilares básicos de la reflexión en docencia.

Las autoras declaran no tener conflicto de interés declarados.

\section{Referencias}

Barrera-Osorio F. \& Linden LL. (2009). The use and misuse of computers in education: evidence from a randomized experiment in Colombia. En World Bank Policy. Research Working Paper.
Jason, J. (2002). Computer and Education 38, 272-285.

Raman R, Sairam B, Veena G, Hardik V. \& Prema N. (2021). Adoption of online proctored examinations by university students during COVID-19. Innovation diffusion study. Educ Inf Techno 31, 1-20.

Reyes-García C, Sosa-Moreno F, Marchena-Gómez R. \& Marchena-Gómez J. (2013). Sistema de evaluación en las prácticas clínicas de la titulación de Medicina. FEM: Revista de la Fundación Educación Médica 16, 7-11.

Valtonen T, Kukkonen J, Dillon P, Väisänen P. \& Hacklin S. (2011). Confronting the technological pedagogical knowledge of Finnish Net Generation student teachers. Technology, Pedagogy and Education 20, 3-18.

Vázquez-Cano E. \& Sevillano M. (2015). Dispositivos digitales móviles en Educación: el aprendizaje ubicuo. Narcea Ediciones. España. 